\title{
JOURNAL OF ENGLISH AS A LINGUA FRANCA
}

\section{INCOMING EDITOR}

Martin Dewey

King's College London, UK

E-mail: martin.dewey@kcl.ac.uk

OUTGOING EDITOR

Barbara Seidlhofer

University of Vienna, Austria

E-mail: barbara.seidlhofer@univie.ac.at

\section{REVIEW EDITOR}

Philip Riley

Rue Félix Bovie 41

1050 Bruxelles

Belgium

E-mail: philip.riley@univ-lorraine.fr

EDITORIAL ASSISTANT

Judith Brockmann

University of Vienna, Austria

\section{DE GRUYTER MOUTON}




\section{EDITORIAL BOARD}

Martin Dewey, King's College,

London, UK

David Deterding, Universiti Brunei

Darussalam

Susanne Ehrenreich, Technische

Universität Dortmund, Germany

Susan Gal, The University of Chicago, USA

Gibson Ferguson, The University of Sheffield, UK

Nobuyuki Honna, Aoyama Gakuin

University, Japan

Juliane House, Universität Hamburg, Germany

Jennifer Jenkins, University of

Southampton, UK
Andy Kirkpatrick, Griffith University, Australia

Anna Mauranen, University of Helsinki, Finland

Tim McNamara, The University of Melbourne, Australia

Salikoko Mufwene, The University of Chicago, USA

Marie-Luise Pitzl, University of Vienna, Austria

Wen Qiufang, Beijing Foreign Studies University, China

Henry Widdowson, University of Vienna, Austria 
ABSTRACTED/INDEXED IN Baidu Scholar · Celdes · Clarivate Analytics: Emerging Sources Citation Index · CNKI Scholar (China National Knowledge Infrastructure) · CNPIEC · De Gruyter: IBR (International Bibliography of Reviews of Scholarly Literature in the Humanities and Social Sciences); IBZ (International Bibliography of Periodical Literature in the Humanities and Social Sciences) · EBSCO (relevant databases) · EBSCO Discovery Service · Elsevier: SCOPUS · Genamics JournalSeek · Google Scholar · J-Gate · JournalTOCs · KESLI-NDSL (Korean National Discovery for Science Leaders) $\cdot$ Linguistic Bibliography Online $\cdot$ Linguistics Abstracts Online $\cdot$ MLA International Bibliography · Naviga (Softweco) · Primo Central (ExLibris) · ProQuest (relevant databases) · ReadCube $\cdot$ ResearchGate $\cdot$ SCImago (SJR) $\cdot$ Sherpa/RoMEO $\cdot$ Summon (Serials Solutions/ ProQuest) · TDNet · Ulrich's Periodicals Directory/ulrichsweb · WanFang Data · WorldCat (OCLC)

The publisher, together with the authors and editors, has taken great pains to ensure that all information presented in this work reflects the standard of knowledge at the time of publication. Despite careful manuscript preparation and proof correction, errors can nevertheless occur. Authors, editors and publisher disclaim all responsibility for any errors or omissions or liability for the results obtained from the use of the information, or parts thereof, contained in this work.

ISSN 2191-9216 · e-ISSN 2191-933X

All information regarding notes for contributors, subscriptions, Open access, back volumes and orders is available online at www.degruyter.com/journals/jelf

RESPONSIBLE EDITOR Barbara Seidlhofer, University of Vienna, Department of English, Universitätscampus AAKH, Spitalgasse 2, Vienna, Austria. E-mail: barbara.seidlhofer@univie.ac.at

JOURNAL MANAGER Denis Fracalossi, De Gruyter, Genthiner Straße 13, 10785 Berlin, Germany, Tel: +49 (0)30 260 05-283, Fax: +49 (0)30 260 05-250.

E-mail: denis.fracalossi@degruyter.com

RESPONSIBLE FOR ADVERTISEMENTS Claudia Neumann, De Gruyter, Genthiner Straße 13, 10785 Berlin, Germany, Tel.: +49 (0)30 260 05-226, Fax: +49 (0)30 260 05-264.

E-mail: anzeigen@degruyter.com

(C) 2019 Walter de Gruyter GmbH, Berlin/Boston

TYPESETtING Integra Software Services Pvt. Ltd., Pondicherry, India.

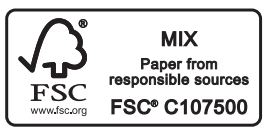


Offenlegung der Inhaber und Beteiligungsverhältnisse gem. § 7a Abs. 1 Ziff. 1, Abs. 2 Ziff. 3 des Berliner Pressegesetzes: Die Gesellschafter der Walter de Gruyter GmbH sind: Cram, Gisela, Rentnerin, Berlin; Cram, Elsbeth, Pensionärin, Rosengarten-Alvesen; Cram, Dr. Georg-Martin, Unternehmens-Systemberater, Stadtbergen; Cram, Maike, Wien (Österreich); Cram, Jens, Mannheim; Cram, Ingrid, Betriebsleiterin, Tuxpan/Michoacan (Mexiko); Cram, Sabina, Mexico, DF (Mexiko); Cram, Silke, Wissenschaftlerin, Mexico DF (Mexiko); Cram, Björn, Aachen; Cram, Berit, Hamm; Cram-Gomez, Susana, Mexico DF (Mexiko); Cram-Heydrich, Walter, Mexico DF (Mexico); Cram-Heydrich, Kurt, Angestellter, Mexico DF (Mexico); Duvenbeck, Brigitta, Oberstudienrätin i.R., Bad Homburg; Gädeke, Gudula, M.A., Atemtherapeutin/Lehrerin, Tübingen; Gädeke, Martin, Einzelunternehmer, Ingolstadt; Gomez Cram, Arturo Walter, Global Key Account Manager, Bonn, Gomez Cram, Ingrid Arlene, Studentin, Mexico, DF (Mexiko), Gomez Cram, Robert, Student, Philadelphia, PA, USA, Lubasch, Dr. Annette, Ärztin, Berlin; Schütz, Dr. Christa, Ärztin, Mannheim; Schütz, Sonja, Berlin; Schütz, Juliane, Berlin; Schütz, Antje, Berlin; Schütz, Valentin, Mannheim; Seils, Dorothee, Apothekerin, Stuttgart; Seils, Gabriele, Journalistin, Berlin; Seils, Christoph, Journalist, Berlin; Siebert, John-Walter, Pfarrer, Oberstenfeld; Tran, Renate, Mediatorin, Zürich (Schweiz). 


\section{Contents}

\section{Editorials}

Barbara Seidlhofer

My JELF Odyssey - 1

Martin Dewey

An ELF-ography - 5

\section{Articles}

Takanori Sato, Yuri Jody Yujobo, Tricia Okada and Ethel Ogane

Communication strategies employed by low-proficiency users: Possibilities for ELF-informed pedagogy — 9

Stefan Bulatović, Anja Schüppert and Charlotte Gooskens

Receptive multilingualism versus ELF: How well do Slovenes understand

Croatian compared to Croatian speakers' English? - 37

Akiko Otsu

The shifting perception of Japanese BELF users towards English: a case study -67

Päivi likkanen

ELF and migrant categorization at family clinics in Finland -97

Andrew Sewell

"Late dividends of the British Empire": Language ideologies and the native/non-native question in online newspaper comments -125

\section{Book Reviews}

Claudio Schekulin

MacKenzie, lan. Language Contact and the Future of English - 155

Marc Deneire

Sherman, Tamah, and Jiři Nekvapil (eds.). English in Business and Commerce: Interactions and Policies: English in Europe, Volume 5-161 
Jessica Mueller

Rabe, Frank. 2016. Englischsprachiges Schreiben und Publizieren in verschiedenen Fachkulturen: Wie deutschsprachige Forscher mit der Anglisierung der Wissenschaftskommunikation umgehen — 167

Philip Riley

Tieken-Boon van Ostade, Ingrid (ed.). English Usage Guides. History, Advice, Attitudes 173

\section{Editors' Note}

JELF peer reviewers 2012-2018 - a big THANK YOU! — 177 